\title{
Sialolitíase em região sublingual direita: relato de caso
}

Sialolitiasis en la región sublingual derecha: reporte de caso

Sialolithiasis in right sublingual region: case report

Camila Barbosa da SILVA ${ }^{\mathbf{1}}$

Lioney Nobre CABRAL ${ }^{2}$

Tiago Novaes PINHEIRO 3

Antonio Jorge Araújo de VASCONCELOS II

${ }^{1}$ Curso de graduação em Odontologia, Escola Superior de Ciências da Saúde, Universidade do Estado do Amazonas-UEA, 69065-001 Manaus-AM, Brasil ${ }^{2}$ Professor da Escola Superior de Ciências da Saúde, Universidade do Estado do Amazonas-UEA, 69065-001 Manaus-AM, Brasil Doutor em Biotecnologia pela Universidade Federal do Amazonas-UFAM

${ }^{3}$ Professor da Escola Superior de Ciências da Saúde, Universidade do Estado do Amazonas-UEA, 69065-001 Manaus-AM, Brasil Mestre e Doutor em Patologia Bucal pela Faculdade de Odontologia de Bauru, Universidade de São Paulo-USP

4Professor da Escola Superior de Ciências da Saúde, Universidade do Estado do Amazonas-UEA, 69065-001 Manaus-AM, Brasil Mestre em Patologia Bucal pela Universidade do Federal do Amazonas-UFAM

\section{Resumo}

Sialolitíases de pequenas proporções localizadas na porção terminal do ducto podem ser difíceis de serem eliminadas de forma passiva, causando problemas ao paciente como episódios de dor e desconforto, podendo evoluir para complicações como infecção ou retenção mucosa. O presente trabalho relata um caso de sialolitíase de uma paciente de 35 anos. Ao exame clínico constatou-se a presença de uma elevação nodular na região de carúncula sublingual, formato cilíndrico, indolor e móvel à palpação, endurecida, coloração amarelada, aumento de volume sensível no pescoço durante algumas refeições, e evolução de sete meses. Ao exame tomográfico, foi possível observar a presença de uma massa hiperdensa na região de assoalho bucal. Inicialmente foi realizado uma ordenha associada a colocação de cone de guta percha, mas não se obteve sucesso. O tratamento realizado foi a remoção cirúrgica do sialólito sem necessidade de síntese associado ao uso tópico de Mud Oral juntamente com a orientação da ordenha para estimulação do fluxo salivar. A hipótese diagnóstica para esta lesão foi de sialolitíase e a mesma foi confirmada através da análise histopatológica. A paciente, continuou em acompanhamento pósoperatório durante 3 semanas, durante este período, a mesma não desenvolveu nenhuma sintomatologia relevante, sendo dispensada após 21 dias. Portanto, diante das possibilidades clínicas e situação da paciente optou-se por métodos mais conservadores e menos invasivos associados a orientações de cuidados pós-operatórios para que se obtivesse uma rápida recuperação e um bom prognóstico.

Descritores: Cálculos das Glândulas Salivares; Glândula Sublingual; Cirurgia Bucal.

\section{Abstract}

Small sialolithiasis located in the terminal portion of the duct can be difficult to passively eliminate, causing problems to the patient such as episodes of pain and discomfort, and may progress to complications such as infection or mucous retention. The present work reports a case of sialolithiasis from a 35-year-old patient. Clinical examination revealed the presence of a nodular elevation in the region of the sublingual caruncle, cylindrical, painless and movable on palpation, hardened, yellowish in color, an increase in the sensitive volume in the neck during some meals, and an evolution of seven months. Upon tomographic examination, it was possible to observe the presence of a hyperdense mass in the region of the oral floor. Initially, milking associated with placing a gutta percha cone was performed, but it was not successful. The treatment performed was the surgical removal of the sialolith without the need for synthesis associated with the topical use of Oral Mud together with the orientation of milking to stimulate the salivary flow. The diagnostic hypothesis for this lesion was Sialolithiasis and it was confirmed through histopathological analysis. The patient, continued in postoperative follow-up for 3 weeks, during this period, she did not develop any relevant symptoms, being discharged after 21 days. Therefore, given the possibilities clinical conditions and the patient's situation, more conservative and less invasive procedures associated with postoperative care guidelines to obtain a quick recovery and a good prognosis.

Descriptors: Salivary Gland Calculi; Sublingual Gland; Surgery, Oral.

\section{Resumen}

La pequeña sialolitiasis ubicada en la porción terminal del conducto puede ser difícil de eliminar pasivamente, causando problemas al paciente, como episodios de dolor e incomodidad, y puede progresar a complicaciones como infección o retención mucosa. El presente trabajo reporta un caso de sialolitiasis de una paciente de 35 años. El examen clínico reveló la presencia de una elevación nodular en la región del carúnculo sublingual, cilíndrica, indolora y móvil a la palpación, endurecida, de color amarillento, un aumento del volumen sensible en el cuello durante algunas comidas y una evolución de siete meses. Tras el examen tomográfico, fue posible observar la presencia de una masa hiperdensa en la región del piso oral. Inicialmente, se realizó el ordeño asociado con la colocación de un cono de gutapercha, pero no tuvo éxito. El tratamiento realizado fue la extracción quirúrgica del sialolito sin la necesidad de síntesis asociada con el uso tópico de Mud oral junto con la orientación del ordeño para estimular el flujo salival. La hipótesis diagnóstica para esta lesión fue la Sialolitiasis y se confirmó mediante análisis histopatológico. La paciente, continuó en el seguimiento postoperatorio durante 3 semanas, durante este periodo, no desarrolló ningún síntoma relevante, siendo dada de alta después de 21 días. Por lo tanto, en vista de las posibilidades clínicas y la situación del paciente, se eligieron métodos más conservadores y menos invasivos asociados con las pautas de atención postoperatoria para obtener una recuperación rápida y un buen pronóstico.

Descriptores: Cálculos de las Glándulas Salivales; Glándula Sublingual; Cirugía Bucal.

\section{INTRODUÇÃO}

Sialolitos são estruturas calcificadas que se desenvolvem no interior das glândulas salivares ou em seus ductos que se formam pela precipitação e acúmulo de componentes orgânicos e inorgânicos da saliva ${ }^{1}$, ao redor de um ninho de debris que podem incluir um muco espesso, bactérias, células do epitélio do ducto ou corpos estranhos ${ }^{2}$.
Os cálculos salivares, são frequentemente localizados nos ductos das glândulas salivares maiores, sendo a submandibular a mais acometida $(72-95 \%)^{3}$ devido as características químicas da saliva e anatômicas como: comprimento do ducto, trajeto ascendente e tortuoso ${ }^{4}$, seguida da parótida $(4-28 \%)^{3}$, sublingual $(1-2 \%)$ e 
raramente acometendo as glândulas salivares menores ${ }^{5}$.

É considerada a alteração glandular mais comum, sendo que cerca de $50 \%$ dos casos de patologias das glândulas salivares são sialolitíases $^{6}$. A sialolitíase das glândulas salivares pode ocorrer em qualquer faixa etária ${ }^{2}$, porém, ocorre mais comumente em adultos do sexo masculino ${ }^{6}$ entre 30 e 40 anos de idade ${ }^{1}$ e raramente acomete crianças ${ }^{6}$. Sua estimativa é que 12 a cada 1000 adultos da população são afetados ${ }^{7}$, e não apresenta predileção por raça ${ }^{8}$.

Os sialolitos normalmente são unilaterais ocorrendo em apenas uma glândula ${ }^{9}$, podendo ser simples $(75,3 \%)^{8}$ ou múltiplos ${ }^{9}$. O formato dos cálculos pode fornecer informações importantes para definir sua localização de origem $^{10}$. Geralmente, os sialolitos alongados ou de formato cilíndrico encontram-se nos ductos e os arredondados ou de forma ovóide no interior da glândula. Na maioria dos casos, os cálculos salivares são pequenos, podendo medir de 1 a $10 \mathrm{~mm}$, sendo que os maiores de $10 \mathrm{~mm}$ são considerados raros e classificados como gigantes ${ }^{4}$. Clinicamente, os cálculos salivares são consistentes e apresentam coloração geralmente amarelada, dependendo da sua constituição ${ }^{6}$.

A sintomatologia pode variar de acordo com o tamanho e a localização do cálculo ${ }^{11}$, bem como o grau de obstrução e a quantidade de pressão produzida no interior da glândula ${ }^{2}$, podendo ser encontrado um aumento de volume da glândula afetada principalmente durante as refeições, sinais de infecção e longos episódios de dor ${ }^{11}$.

$\mathrm{Na}$ maioria dos casos, os métodos de diagnóstico clínico incluem inspeção, palpação, verificação da quantidade e da qualidade da saliva secretada e exames radiográficos convencionais $^{12}$, mas, em alguns casos, pode-se utilizar técnicas mais avançadas, como sialografia, ultrassonografia, tomografia computadorizada, cintilografia, ressonância magnética e endoscopia, que possibilitam uma melhor localização, mensuração do tamanho do sialolito e consequente planejamento cirúrugico ${ }^{13}$.

As formas de tratamento variam de acordo com o tamanho, formato e localização do sialolito na glândula afetada ${ }^{14}$, podendo ser utilizados desde métodos conservadores como à ordenha e uso de sialogogos, resultando em expulsão instântanea, até tratamentos mais invasivos como remoção cirúrgica intra ou extraoral $^{15}$.

Deste modo, este trabalho irá relatar um caso clínico de sialolitíase em região sublingual que foi tratado da forma mais conservadora possível, com a devida orientação de cuidados pré e pós-operatórios para que se tivesse uma rápida recuperação e um bom prognóstico.

CASO CLÍNICO

Paciente, gênero feminino, 35 anos de idade, melanoderma, foi encaminhada à Clínica de Estomatologia da Universidade do Estado do Amazonas-UEA, queixando-se de elevação nodular em região de assoalho bucal. Durante a anamnese, relatou que o tempo de evolução era de sete meses, acompanhada de sintomatologia dolorosa com aumento de volume sensível na região submandibular direita durante algumas refeições, sendo mais intensa durante a ingestão de frutas e bebidas cítricas.

Ao exame clínico intraoral, observou-se a presença de aumento de volume de coloração amarelada na carúncula sublingual direita, esta localização impossibilitou definir a origem glandular do cálculo. Durante a palpação, constatou-se um nódulo de consistência endurecida e móvel que obstruía a passagem de saliva. Foi utilizada a manobra semiotécnica de ordenha associada a colocação de um cone de guta percha para dilatar o óstio do ducto na tentativa de expelir o sialolito, durante várias tentativas, mas este mostrou-se bastante aderido, inviabilizando a saída de forma passiva e conservadora (Figura 1).

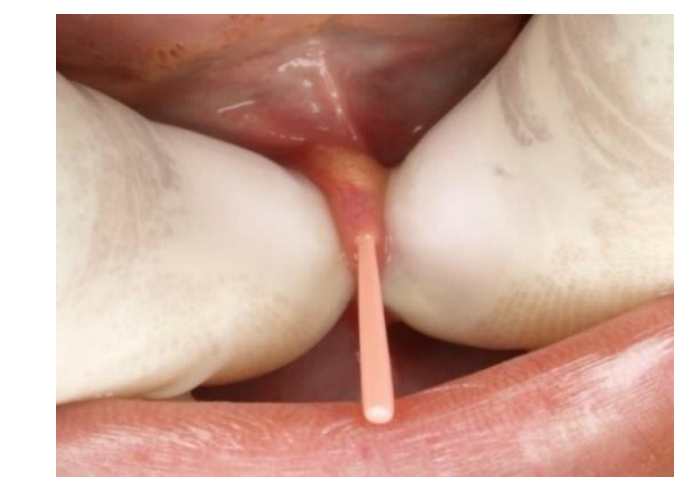

Figura 1: Aspecto inicial da lesão e realização de ordenha.

Foi solicitada, como exame complementar, a tomografia computadorizada na tentativa de se descartar ou confirmar a presença de outros cálculos, esta hipótese foi descartada e verificou-se apenas a presença de uma estrutura hiperdensa na região de carúncula sublingual direita, compatível com apenas um sialolito (Figura 2). Com base nas informações colhidas na anamnese e no achado radiográfico, concluiu-se o diagnóstico clínico de sialolitíase. $O$ tratamento proposto foi a remoção cirúrgica, através de acesso intraoral sob anestesia de bloqueio de nervo lingual bilateral. Uma pequena incisão de aproximadamente $3 \mathrm{~mm}$ foi realizada de forma linear sobre 0 
cálculo, e as paredes do ducto foram divulsionadas cuidadosamente, o que facilitou a visualização e remoção da estrutura calcificada, sem necessidade de síntese (Figura 3). A forma de tratamento estabelecida foi a mais conservadora possível, utilizando incisão simples e exérese por pinça, descartando técnicas traumáticas e mutiladoras à glândula salivar e ao ducto.

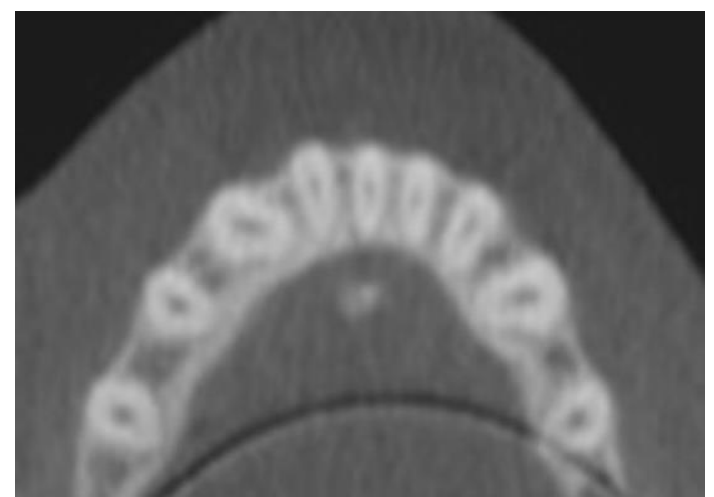

Figura 2: Estrutura hiperdensa no assoalho bucal.

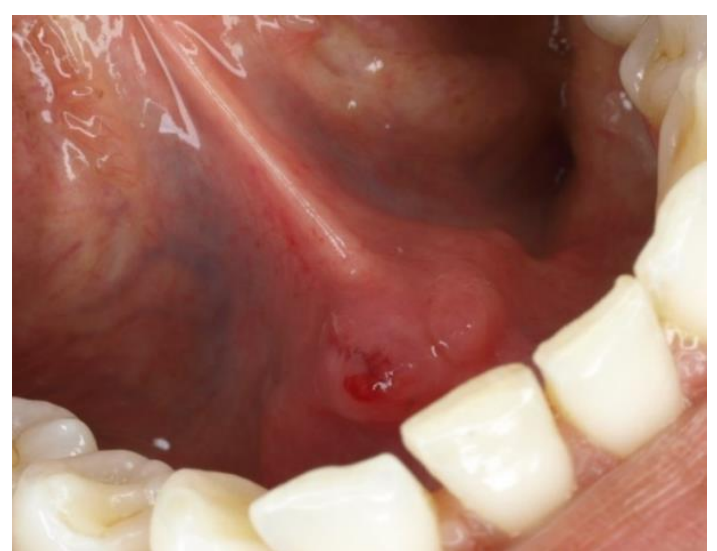

Figura 3: Local da incisão após biópsia excisional.

O material calcificado coletado na biópsia excisional apresentou formato cilíndrico e tamanho de aproximadamente $4 \mathrm{~mm}$ em seu comprimento (Figura 4). O mesmo foi incluído em solução formalina a $10 \%$ e encaminhado ao exame histopatológico.

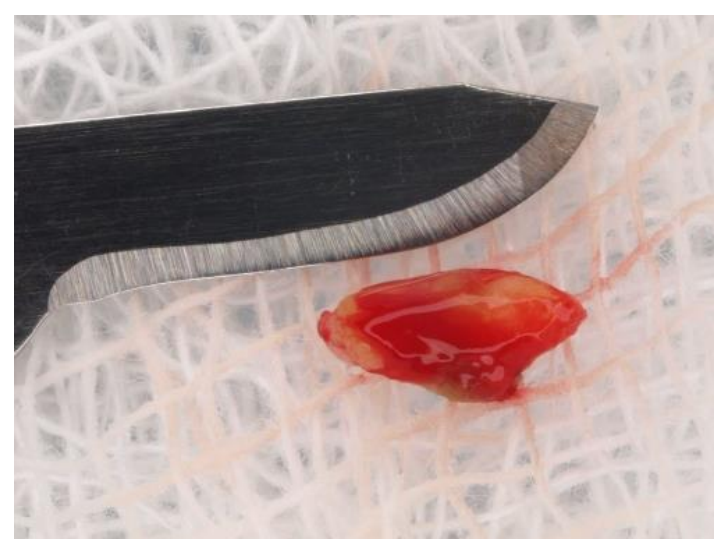

Figura 4: Material calcificado coletado na biópsia excisional.

Foi constatado microscopicamente um fragmento de material mineralizado formado pela deposição apositiva de matéria orgânica tomando aspecto concêntrico e multifocal, e presença de material mucinoso eosinofílico amorfo (Figura 5), que ao ser submetido à microscopia de fluorescência apresentou propriedades fluorescentes, absorvendo e emitindo luz azul (Figura 6).

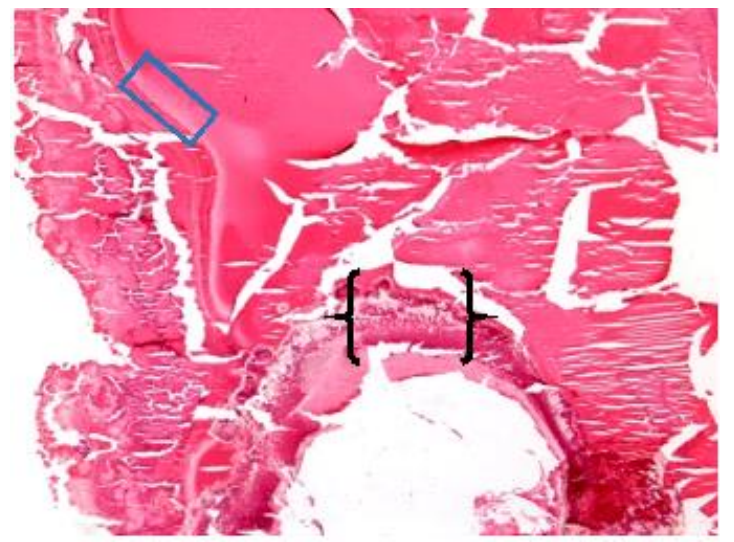

Figura 5: Matéria orgânica concêntrica (em azul) e material mucinoso eosinofílico amorfo (em preto).

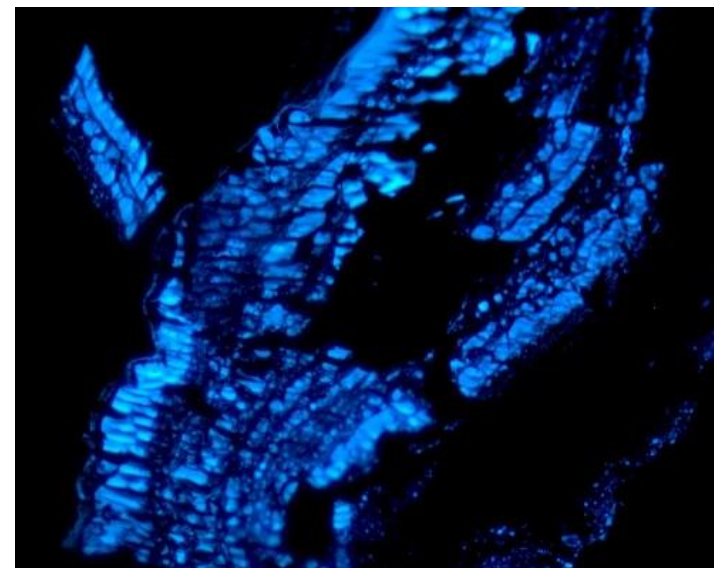

Figura 6: Propriedades fluorescentes do material mucinoso.

A paciente retornou depois de três semanas de pós-operatório, relatando ausência de sintomatologia dolorosa e reorganização das características anatômicas normais da carúncula sublingual, além da retomada do fluxo salivar que estava obstruído antes da cirurgia (Figura 7). No momento encontra-se com sete meses de proservação, estando com salivação normal e sem aumento volumétrico em assoalho bucal (Figura 8 ).

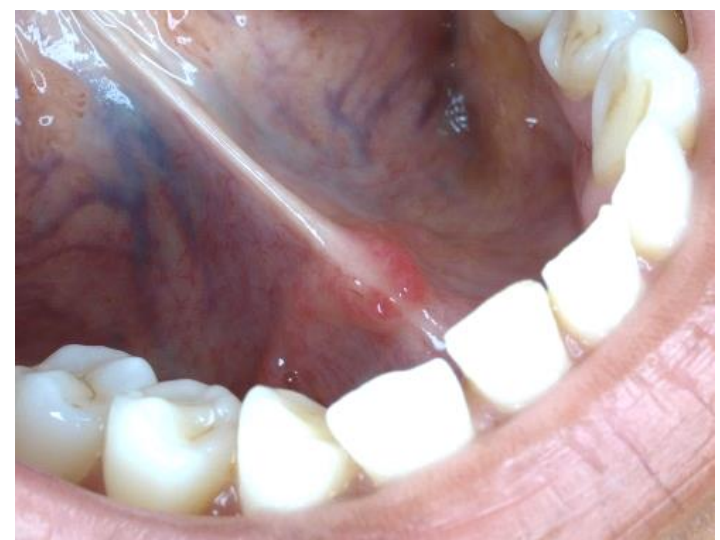

Figura 7: Pós-operatório de três semanas de proservação. 


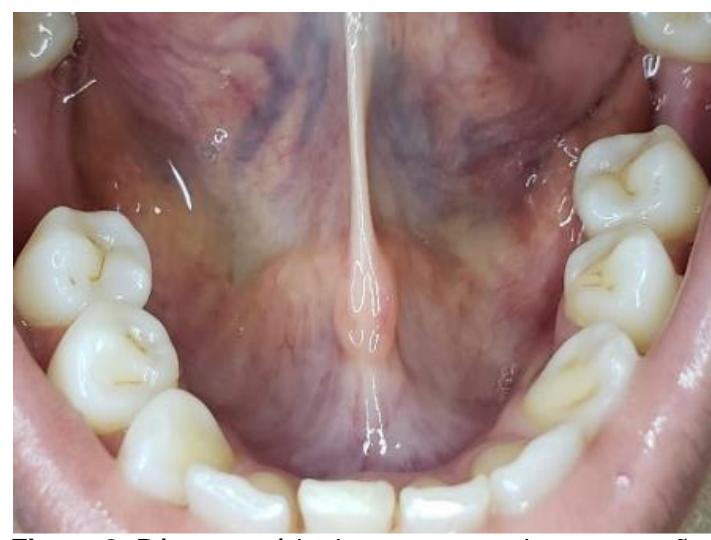

Figura 8: Pós-operatório de sete meses de proservação.

Portanto, diante das possibilidades clínicas e situação da paciente optou-se por métodos mais conservadores e menos invasivos associados a orientações de cuidados pósoperatórios para que se obtivesse uma rápida recuperação e um bom prognóstico.

DISCUSSÃO

A sialolitíase é uma condição patológica comum das glândulas salivares que consiste na formação de concreções calcificadas nos sistemas de ductos ou no interior de uma glândula $^{16}$. A etiologia exata ainda é desconhecida, podendo suas causas serem mecânicas, traumáticas, infecciosas, químicas, neurogênicas ou resultantes da presença de corpo estranho ${ }^{14}$. Quanto ao gênero, há uma incidência maior em homens de 30 a 40 anos de idade $^{10}$, o que difere do caso clínico aqui apresentado.

A glândula submandibular é a mais acometida (80\%), o que pode ser explicado pelo $\mathrm{pH}$ alcalino, secreção mucosa e alta concentração de cálcio desta glândula ${ }^{17}$, haja vista que seu ducto longo e tortuoso, que circunda o músculo milo-hióideo e a secreção no sentido antigravitacional também podem contribuir para a sua predileção ${ }^{18}$. A glândula parótida tem menor prevalência (4-10\%) devido ao auxílio dos músculos da expressão facial no fluxo salivar associado a disposição horizontal do ducto de Stenon ${ }^{19}$, seguida da rara prevalência na glândula sublingual (1-2\%) que deve-se ao menor comprimento dos ductos e ao fato de possuir várias aberturas que favorecem a drenagem da saliva ${ }^{20}$.

Geralmente, os sialolitos são unilaterais ${ }^{9}$ e apresentam coloração amarelada ${ }^{6}$, conforme o do caso clínico em questão. Seu formato está relacionado a sua localização de origem ${ }^{10}$, uma vez que os cálculos de forma arredondada ou ovóide encontram-se no interior da glândula e os de formato cilíndrico ou alongado no interior do ducto ${ }^{4}$, exatamente como o cálculo encontrado na paciente.
A sintomatologia está relacionada com o grau de obstrução, podendo ser assintomática quando a obstrução é parcial e permite a passagem de saliva ${ }^{21,22}$. No entanto, a obstrução completa causa dores e inchaços da glândula durante os períodos de refeições ou quando há estímulos salivatórios ${ }^{6}, 0$ que corrobora com a sintomatologia descrita pela paciente.

A associação de um exame clínico cuidadoso com informações colhidas na anamnese é importante para o diagnóstico desta $\operatorname{condição~}^{18}$, bem como a palpação bimanual na direção anterior e posterior do assoalho bucal ${ }^{6}$, além de exames radiográficos diversos para detectar essas estruturas calcificadas ${ }^{16}$. Em casos onde não foi possível o diagnóstico através de exames ragiográficos, torna-se necessário a utilização de métodos imagenológicos mais precisos como a tomografia computadorizada e a ressonância magnética, apesar de seu alto custo $^{23}$. Nesse caso, optou-se pela tomografia computadorizada disponibilizada na policlínica da UEA sem gerar custos à paciente, uma vez que o sialolito não foi evidenciado na radiografia oclusal.

Para os sialólitos de pequeno tamanho e localizados próximos aos óstios do ducto, o tratamento pode ser conservador, através da ordenha ou pelo cateterismo e a dilatação do ducto $^{1}$, além do aumento na ingestão de líquidos e bebidas cítricas para expulsar o sialolito $^{14}$. Entretanto, esses métodos não foram efetivos no caso clínico em questão, uma vez que o sialolito mostrou-se bastante aderido ao ducto, e assim, optou-se por remoção cirúrgica com uma mínima incisão linear sobre o cálculo para remoção do sialolito, sem utilização de síntese para preservar a integridade do ducto e recuperar o fluxo salivar que estava obstruido.

CONCLUSÃO

Portanto, diante das possibilidades clínicas e situação da paciente, optou-se por métodos de tratamentos os mais conservadores possíveis e menos invasivos associados a orientações de cuidados pós-operatórios para que se obtivesse uma rápida recuperação e um bom prognóstico. Durante sete meses de acompanhamento pós-operatório, a paciente relata ausência de sintomatologia dolorosa, e o local da remoção cirúrgica apresenta reorganização das características anatômicas normais da carúncula sublingual, além da retomada do fluxo salivar.

REFERÊNCIAS

1. Silveira $R L$, Machado $R$, Borges $H$, Oliveira $R$. 
Múltiplos sialolitos em glândula submandibular direita: relato de caso. Rev Fac Oontol Lins. 2005;17(1):39-42.

2. Neville BW, Damm DD, Allen CM, Bouquot JE. Patologia Oral e Maxilofacial. 3. ed., Rio de Janeiro: Elsevier; 2009.

3. Kraaij S, Karagozoglu $\mathrm{KH}$, Forouzanfar $\mathrm{T}$, Veerman EC, Brand HS. Salivary stones: symptoms, aetiology, biochemical composition and treatment. Br Dent J. 2014;217(11):E23.

4. Parkar MI, Vora MM, Bhanushali DH. A Large Sialolith Perforating the Wharton's Duct: Review of Literature and a Case Report. J Maxillofac Oral Surg. 2012;11(4):477-82.

5. Lustmann J, Regev E, Melamed Y. Sialolithiasis. A survey on 245 patients and a review of the literature. Int $\mathrm{J}$ Oral Maxillofac Surg. 1990;19(3):135-38.

6. Landgraf $H$, Assis AFD, Klüppel LE, Oliveira CFD, Gabrielli MAC. Extenso sialolito no ducto da glândula submandibular: relato de caso. Rev Cir Traumatol Buco-Maxilo-Fac. 2006;6(2): 29-34.

7. Iqbal A, Gupta AK, Natu SS, Gupta AK. Unusually large sialolith of Wharton's duct. Ann Maxillofac Surg. 2012;2(1):70-3.

8. Hazarika P, Punnoose SE, Singh R, Arora S. Deep and unusual sialolithiasis of submandibular duct and gland: a surgical dilemma. Indian J Otolaryngol Head Neck Surg. 2013;65(4):309-13.

9. Choi J, Kim IK, Oh NS. Multiple sialoliths in sublingual gland: report of a case. Int $\mathrm{J}$ Oral Maxillofac Surg. 2002;31(5):562-63.

10. Jaeger F, Andrade R, Alvarenga RL, Galizes $\mathrm{BF}$, Amaral MBF. Sialolito gigante no ducto da glândula submandibular. Rev Port Estomatol Med Dent Cir Maxilofac. 2013;54(1):33-6.

11. Sobrino-Guijarro B, Cascarini L, Lingam RK. Advances in imaging of obstructed salivary glands can improve diagnostic outcomes. Oral Maxillofac Surg. 2013;17(1):11-9.

12. Austin T, Davis J, Chan T. Sialolithiasis of submandibular gland. J Emerg Med. 2004;26(2):221-23.

13. Mandel L, Alfi D. Diagnostic imaging for submandibular duct atresia: literature review and case report. J Oral Maxillofac Surg. 2012;70(12):2819-22.

14. Manzi FR, Silva AIV, Dias FG, de Fátima Ferreira E. Sialolito na glândula submandibular: Relato de caso clínico. ROBRAC. 2010;19(50): 270-74.

15. Singh Gill MP, SINGH AP. Spontaneous expulsion of an asymptomatic large submandibular salivary gland calculus: a case report. JCDR. 2012; 6(5):908-9.

16. Farenzena KP, da Rocha Vieira $R$, Mallmann CT, De Carli JP, da Silva SO. Sialolitíase: revisão de literatura e levantamento de casos. Odonto. 2012;20(40):31-9.

17. Graziani F, Vano M, Cei S, Tartaro G, Mario G. Unusual asymptomatic giant sialolith of the submandibular gland: a clinical report. J Craniofac Surg. 2006;17(3):549-52.

18. Siddiqui SJ. Sialolithiasis: an unusually large submandibular salivary stone. $\mathrm{Br}$ Dent $\mathrm{J}$. 2002;193(2):89-91.

19. Arunkumar KV, Garg N, Kumar V. Oversized submandibular gland sialolith: a report of two cases. J Maxillofac Oral Surg. 2015; 14(Suppl 1):116-19.

20.Su YX, Zhang K, Ke ZF, Zheng GS, Chu M, Liao GQ. Increased calcium and decreased magnesium and citrate concentrations of submandibular/sublingual saliva in sialolithiasis. Arch oral biol. 2010;55(1):15-20.

21. Magnabosco Neto AE. Sialolito na regiäo de glândula parótica: relato de um caso clínico. BCl. 2002;9(35):210-14.

22. Pereira RS, Bonardi JP, Silva JR, Mourão CFAB, Souza FI, Pimentel RM. Remoção cirúrgica de sialolito no ducto da glândula submandibular: relato de caso. Arch Health Invest (2017);6(4):185-88.

23. Silva ARS, Soares GR, Soubhia AMP, Miyahara GI. Considerações atuais da sialolitíase de ducto de glândula submandibular. Rev Odontol Araçatuba. 2010;46-50.

\section{CONFLITO DE INTERESSES}

Os autores declaram não haver conflitos de interesse

AUTOR PARA CORRESPONDÊNCIA

\section{Camila Barbosa da Silva}

Rua do Oriente, $\mathrm{n}^{\circ} 10$, Bairro Parque Mauá

69075-722 Manaus-AM, Brasil

E-mail: cbs.odo16@uea.edu.br 